\title{
The long noncoding RNA GAS5 negatively regulates the adipogenic differentiation of MSCs by modulating the miR-18a/CTGF axis as a ceRNA
}

\author{
Ming Li', Zhongyu Xie ${ }^{1}$, Peng Wang ${ }^{1}$, Jinteng Li', Wenjie Liu', Su'an Tang ${ }^{1}$, Zhenhua Liư ${ }^{2}$ Xiaohua Wu ${ }^{3}$,
} Yanfeng $\mathrm{Wu}^{3}$ and Huiyong Shen ${ }^{1}$

\begin{abstract}
Mesenchymal stem cells (MSCs) are important pluripotent stem cells and a major source of adipocytes in the body. However, the mechanism of adipogenic differentiation has not yet been completely elucidated. In this study, the long noncoding RNA GAS5 was found to be negatively correlated with MSC adipogenic differentiation. GAS5

overexpression negatively regulated adipocyte formation, whereas GAS5 knockdown had the opposite effect. Further mechanistic analyses using luciferase reporter assays revealed that GAS5 regulates the adipogenic differentiation of MSCs by acting as competing endogenous RNA (ceRNA) to sponge miR-18a, which promotes adipogenic

differentiation. Mutation of the binding sites for GAS5 in miR-18a abolished the effect of the interaction. The miR-18a mimic and inhibitor reversed the negative regulatory effect of GAS5 on MSCs adipogenic differentiation. In addition, GAS5 inhibited miR-18a, which downregulates connective tissue growth factor (CTGF) expression, to negatively regulate the adipogenic differentiation of MSCs. Taken together, the results show that GAS5 serves as a sponge for miR-18a, inhibiting its capability to suppress CTGF protein translation and ultimately decreasing the adipogenic differentiation of MSCs. GAS5 is an important molecule involved in the adipogenic differentiation of MSCs and may contribute to the functional regulation and clinical applications of MSCs.
\end{abstract}

\section{Introduction}

Mesenchymal stem cells (MSCs) are stem cells with selfrenewal and multi-potential differentiation abilities, which allow them to differentiate into osteogenic, adipogenic, and chondrogenic lineages ${ }^{1}$. MSCs have been used in numerous clinical applications due to their advantageous characteristics, including their ability to be easily derived

\footnotetext{
Correspondence: Yanfeng Wu (wuyanfengcn@126.com) or

Huiyong Shen (shenhuiy@yeah.net)

'Department of Orthopedics, Sun Yat-sen Memorial Hospital, Sun Yat-sen University, Guangzhou 510120, People's Republic of China

2Department of Orthopedics, Zhujiang Hospital, Southern Medical University, Guangzhou 510120, People's Republic of China

Full list of author information is available at the end of the article.

These authors contributed equally: Ming Li, Zhongyu Xie, Peng Wang

Edited by A. Stephanou
}

from many sources ${ }^{2}$. For applications involving adipogenic differentiation, MSCs have been used in breast augmentations $^{3}$ and implanted subcutaneously into soft-tissue defects $^{4}$. As the main source of adipocytes, an understanding of the specific mechanism through which MSCs undergo adipogenic differentiation is of great importance. Previous studies have identified some factors that play roles in the adipogenic differentiation of $\mathrm{MSCs}^{5,6}$. However, the specific mechanisms have not yet been completely elucidated, and further research is needed.

Noncoding RNAs are a type of non-protein coding RNA that critically regulate processes determining cell fate. Long noncoding RNAs (lncRNAs) are transcripts containing $>200$ nucleotides of RNA that do not encode

\section{(c) The Author(s) 2018}

(c) (i) Open Access This article is licensed under a Creative Commons Attribution 4.0 International License, which permits use, sharing, adaptation, distribution and reproduction in any medium or format, as long as you give appropriate credit to the original author(s) and the source, provide a link to the Creative Commons license, and indicate if changes were made. The images or other third party material in this article are included in the article's Creative Commons license, unless indicated otherwise in a credit line to the material. If material is not included in the article's Creative Commons license and your intended use is not permitted by statutory regulation or exceeds the permitted use, you will need to obtain permission directly from the copyright holder. To view a copy of this license, visit http://creativecommons.org/licenses/by/4.0/. 
proteins. According to some recent studies, lncRNAs affect MSCs differentiation. For instance, HOTAIR impacts MSCs differentiation and is related to senescence-associated DNA methylation ${ }^{7}$. However, the main determinants that influence adipocyte formation by MSCs remain unclear. The lncRNA growth arrest-specific transcript 5 (GAS5) is a non-protein coding RNA that is considered a tumor suppressor gene $e^{8,9}$. GAS5 exerts crucial effects on biological proliferation and differentiation $^{10-12}$, but researchers have not determined whether GAS5 affects the adipogenic differentiation of MSCs.

MicroRNAs are another type of noncoding RNA that mainly binds to the 3' UTR of target mRNAs to inhibit protein translation ${ }^{13}$. MicroRNAs are important regulators of MSCs differentiation ${ }^{14,15}$. In the most recent studies, lncRNAs were shown to act as competing endogenous RNAs (ceRNAs) to sponge miRNAs, thus regulating cell differentiation and other functions ${ }^{16,17}$. For example, GAS5 sponges miR-222 to function as a tumor suppressor in human glioma cells ${ }^{9}$, and MEG3 functions as a ceRNA to regulate ischemic neuronal death by targeting the miR-21/PDCD4 signaling pathway ${ }^{18}$. However, studies confirming that GAS5 acts as ceRNA in MSCs have not yet been reported.

MicroRNA-18a is an important member of the miR-1792 cluster that plays a carcinogenic role in some tumors ${ }^{19-21}$ and is important for differentiation and apoptosis $^{22,23}$. Connective tissue growth factor (CTGF), a member of a family of cysteine-rich matricellular proteins, recently emerged as a multifunctional regulator because it controls diverse cellular processes, as well as vascular and skeletal development ${ }^{24-26}$. In some studies, the biological function of miR-18a has been reported to be mediated by its binding to the $3^{\prime}$ UTR of CTGF $^{27}$. In addition, some reports of leukemia bone marrow engraftment have indicated that CTGF inhibits the differentiation of MSCs into adipocytes ${ }^{28}$. However, researchers have not clearly determined whether the micro18a/CTGF axis participates in the adipogenic differentiation of MSCs.

Our research illuminates the mechanism through which GAS5 affects the adipogenic differentiation of MSCs. GAS5 negatively regulated adipocyte differentiation in a miR-18a/CTGF-dependent manner, and GAS5 knockdown promoted the differentiation of MSCs into adipocytes. Our findings improve our understanding of the mechanisms of MSCs adipogenic differentiation and may contribute to future molecular therapies using MSCs.

\section{Methods}

\section{Cell isolation and culture}

This study was approved by the ethics committee of Sun Yat-sen Memorial Hospital at Sun Yat-sen University (Guangzhou, People's Republic of China). Eighteen healthy donors aged 20-30 years were selected for the study.
Bone marrow was extracted from the posterior superior iliac spine under sterile conditions. MSCs were purified and isolated using our previously reported methods ${ }^{29}$. MSCs at passage 3 to 5 were used in the experiments. Cells were seeded in 12-well plates and cultured in Dulbecco's Modified Eagle's Medium (DMEM; Gibco, New York, USA) containing $10 \%$ fetal bovine serum (FBS; Gibco) at $37^{\circ} \mathrm{C}$ in a $5 \% \mathrm{CO}_{2}$ atmosphere. The medium was replaced every 3 days, and MSCs were passaged to $90 \%$ confluence.

\section{Multipotent differentiation potential of MSCs Adipogenic differentiation}

For adipogenic induction, MSCs were cultured in adipogenic medium consisting of DMEM supplemented with 10\% FBS, $0.5 \mathrm{mM}$ 3-isobutyl-1-methylxanthine (SigmaAldrich, St. Louis, USA), $1 \mathrm{mM}$ dexamethasone (SigmaAldrich), $10 \mathrm{mg} / \mathrm{ml}$ insulin (Sigma-Aldrich), $0.2 \mathrm{mM}$ indomethacin (Sigma-Aldrich), $100 \mathrm{IU} / \mathrm{ml}$ penicillin (Sigma-Aldrich), and $100 \mathrm{IU} / \mathrm{ml}$ streptomycin (SigmaAldrich). The medium was replaced every 3 days. The cells were stained with ORO on day 14.

\section{Osteogenic differentiation}

MSCs were seeded in 12-well plates and cultured in osteogenic differentiation medium for 0-21 days. This medium was composed of DMEM containing 10\% FBS, $0.1 \mu \mathrm{M}$ dexamethasone, $10 \mathrm{mM} \beta$-glycerol phosphate, 50 $\mu \mathrm{M}$ ascorbic acid (Sigma-Aldrich), $100 \mathrm{IU} / \mathrm{ml}$ penicillin (Sigma-Aldrich), and $100 \mathrm{IU} / \mathrm{ml}$ streptomycin (SigmaAldrich). The medium was replaced every 3 days. The cells were stained with Alizarin Red S on day 14.

\section{Chondrogenic differentiation}

MSCs were grown as high-density pellets $\left(5 \times 10^{5}\right.$ cells) for 3 weeks in specific medium. Serum-free chondrogenic medium containing high-glucose DMEM was supplemented with 1\% ITS Premix (Corning Life Sciences, Wisconsin, USA), $50 \mathrm{mg} / \mathrm{l}$ ascorbic acid, $1 \mathrm{mM}$ sodium pyruvate (Sigma-Aldrich), $100 \mathrm{nM}$ dexamethasone, and $10 \mathrm{ng} / \mathrm{ml}$ recombinant human TGF- $\beta 3$ (R\&D Systems, Minnesota, USA). On day 21, the pellets were prepared for histology and stained with toluidine blue to detect the secretion of sulfated glycosaminoglycans.

\section{Oil Red O (ORO) staining and quantification}

ORO was dissolved in isopropyl alcohol at a concentration of $0.5 \%$ as the stock solution. The stock solution was diluted with ultrapure water at a ratio of 3:2 to obtain the working solution. MSCs were fixed with $4 \%$ paraformaldehyde and stained with ORO in the working solution for $15 \mathrm{~min}$. After three washes, the stained cells were observed under a microscope and visualized in photomicrographs. The stained cells were destained with 
isopropyl alcohol. A 200- $\mu \mathrm{l}$ aliquot was transferred to a 96-well plate, and the absorbance at $520 \mathrm{~nm}$ was measured.

\section{Flow cytometry}

MSCs were digested with $0.25 \%$ trypsin supplemented with $0.53 \mathrm{mM}$ EDTA (Gibco). After centrifugation, MSCs were resuspended in phosphate-buffered saline (PBS) and incubated for $30 \mathrm{~min}$ with antibodies against human CD29-phycoerythrin (PE), CD34-allophycocyanin (APC), CD44-fluorescein isothiocyanate (FITC), CD45-FITC, CD105-FITC, or HLA-DR-PE. All of the antibodies used in flow cytometry are purchased from BD Biosciences (New York, USA). Flow cytometry was performed to identify the MSC phenotypes. For the cell cycle analysis, MSCs were trypsinized and fixed with $80 \%$ cold alcohol at $4{ }^{\circ} \mathrm{C}$ for $12 \mathrm{~h}$. After centrifugation, MSCs were resuspended in $50 \mu \mathrm{l}$ of RNase and $450 \mu \mathrm{l}$ of propidium iodide (PI; Sigma-Aldrich) staining fluid, and the phases of the cell cycle in each sample after 30 min of incubation were analyzed by flow cytometry.

\section{Lentivirus construction and infection}

The IncRNA GAS5 and CTGF siRNA were designed and synthesized by GenePharma (Shanghai, China). We selected one siRNA (Supplementary Table 1) with the best knockdown efficiency to build the short hairpin RNA (shRNA) and construct the lentivirus. The lncRNA GAS5 wild type (WT) and lncRNA GAS5 mutant type (MUT) overexpression lentiviruses were generated by cotransfecting pGLVH1/GFP/Puro (Gene Pharma) and packing plasmids (pGag/Pol, pRev, and pVSV-G) into $293 \mathrm{~T}$ cells. Culture supernatants containing lentiviruses were filtered and concentrated $72 \mathrm{~h}$ after transfection. Each lentivirus $\left(10^{9}\right.$ transducing units $\left./ \mathrm{ml}\right)$ and polybrene $(5 \mathrm{mg} / \mathrm{ml})$ were added to the medium, and MSCs were incubating with the resulting mixture for $24 \mathrm{~h}$ at a multiplicity of infection of 50. In both the overexpression and downregulation experiments, we infected MSCs with the lentivirus on day 0 , removed the lentivirus-containing medium after $24 \mathrm{~h}$, and then began to induce adipogenic differentiation. Related experiments were performed using induced MSCs.

\section{Real-time quantitative reverse transcription-polymerase chain reaction (qRT-PCR)}

MSCs were cultured in 12-well plates. TRIzol (Invitrogen, Massachusetts, USA) was added to the wells at the appropriate times, and total RNA was isolated from the MSCs and transcribed into complementary DNAs using a PrimeScript RT reagent kit (TaKaRa, Dalian, China). qRTPCR was then performed with a LightCycler 480 PCR system (Roche, Basel, Switzerland) using SYBR Premix Ex Taq (TaKaRa). The detailed method was described in our previously published studies ${ }^{30}$. The results were first normalized to the level of GAPDH expression. The data were standardized based on GAPDH expression, and the $2^{-\Delta \Delta \mathrm{Ct}}$ method was used to analyze the data and determine the relative expression of each gene. The primers for miRNA were provided by Guangzhou Ribobio Biotechnology Co., Ltd. The miRNA RT reaction was based on primers with a stem loop structure. The reverse transcription primers for the stem loop structure bind to the 3 '-end of the miRNA, and the reverse transcription reaction was performed using reverse transcriptase. Specific forward primers, universal reverse primers, and SYBR Green fluorescent dyes for quantitative amplification were used to enable the real-time quantitative detection of reverse transcription products. The forward and reverse primers for each gene are listed in Supplementary Table 2.

\section{Western blot and agarose gel electrophoresis}

MSCs were lysed at the appropriate times and centrifuged at $14,000 \mathrm{rpm} / \mathrm{min}$, and the proteins were then boiled with loading buffer. Equal amounts of protein extracts were separated on 10 or $12 \%$ gels and transferred to polyvinylidene fluoride (PVDF) membranes (Millipore, Massachusetts, USA). The membranes were blocked with BSA and incubated with primary antibodies against AGO2 (Abcam, Cambridge, United Kingdom), PPAR- $\gamma$ (Cell Signaling Technology, Massachusetts, USA), C/EBP$\alpha$ (Abcam), FABP4 (Abcam), CTGF (R\&D Systems) and GAPDH (Beyotime, Shanghai, China) overnight at $4{ }^{\circ} \mathrm{C}$. After three washes, the PVDF membranes were incubated with a horseradish peroxidase (HRP)-conjugated secondary antibody (diluted 1:3000; Santa Cruz Biotechnology), and specific antibody-antigen complexes were detected using Immobilon Western Chemiluminescent HRP Substrate (Millipore).

PCR products were collected in microtubes with DNA loading buffer and separated on a $1.2 \%$ agarose containing $0.01 \%$ Gel red (Biotium). DNA products were detected by UV light and photographed for observation.

\section{Isolation of cytoplasmic and nuclear fractions from cells}

Cytoplasmic and nuclear RNA was extracted from MSCs using the PARIS ${ }^{\mathrm{m}}$ Kit (Thermo Scientific), according to the manufacturer's instructions. The RNAs extracted from each of the fractions were analyzed by qRT-PCR. The data were analyzed to determine the nuclear and cytoplasmic levels of each RNA.

\section{Luciferase reporter assay}

The lncRNA GAS5 WT and IncRNA GAS5 MUT sequences were synthesized and cloned into pmirGLO plasmid vectors, and the constructed vectors were transfected into MSCs with Lipofectamine 3000 Transfection 
Reagent (Invitrogen). The miRNA mimic or negative control was added to specific wells of the plate. Relative luciferase activities were measured using the Dual-Luciferase $^{\circledR}$ Reporter $\left(D^{2} R^{\mathrm{st}}\right.$ ) Assay System (Promega, Wisconsin, USA) according to the manufacturer's instructions and analyzed as the ratio of firefly luciferase activity to Renilla luciferase activity.

\section{RNA-binding protein immunoprecipitation}

The Magna RIP ${ }^{\mathrm{mx}}$ RNA-Binding Protein Immunoprecipitation Kit (Millipore) was used for the RIP assay. Briefly, about $5 \times 10^{6}$ of MSCs was lysed with RIP lysis buffer and then incubated with RIP buffer containing magnetic beads conjugated with the anti-AGO2 antibody or negative control purified rabbit IgG. A positive control antiSNRNP70 antibody was used for the RIP procedure. Samples were incubated with proteinase $\mathrm{K}$, and immunoprecipitated RNAs were isolated. The precipitated RNAs were purified and subjected to quantitative PCR to detect the presence of the target lncRNA or miRNA.

\section{RNA pull-down assay}

A Pierce $^{\text {Tm }}$ Magnetic RNA-Protein Pull-Down Kit (Thermo Fisher Scientific, Massachusetts, USA) was used for the RNA pull-down assay. Biotin-labeled RNAs were transcribed in vitro with the TranscriptAid T7 High-Yield Transcription Kit (Thermo Fisher Scientific) and Pierce RNA 3' End Desthiobiotinylation Kit (Thermo Fisher

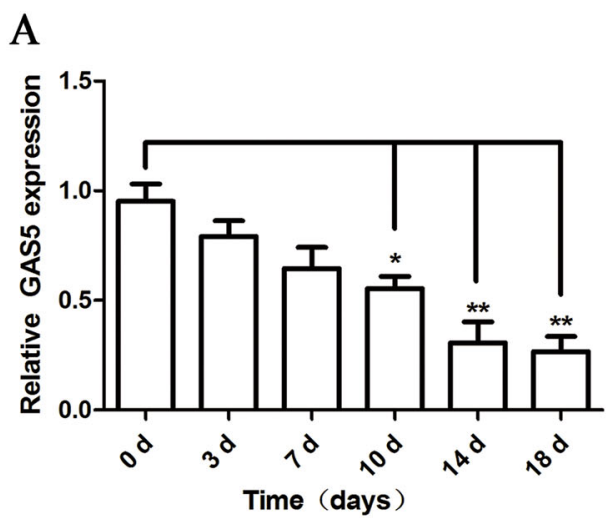

C
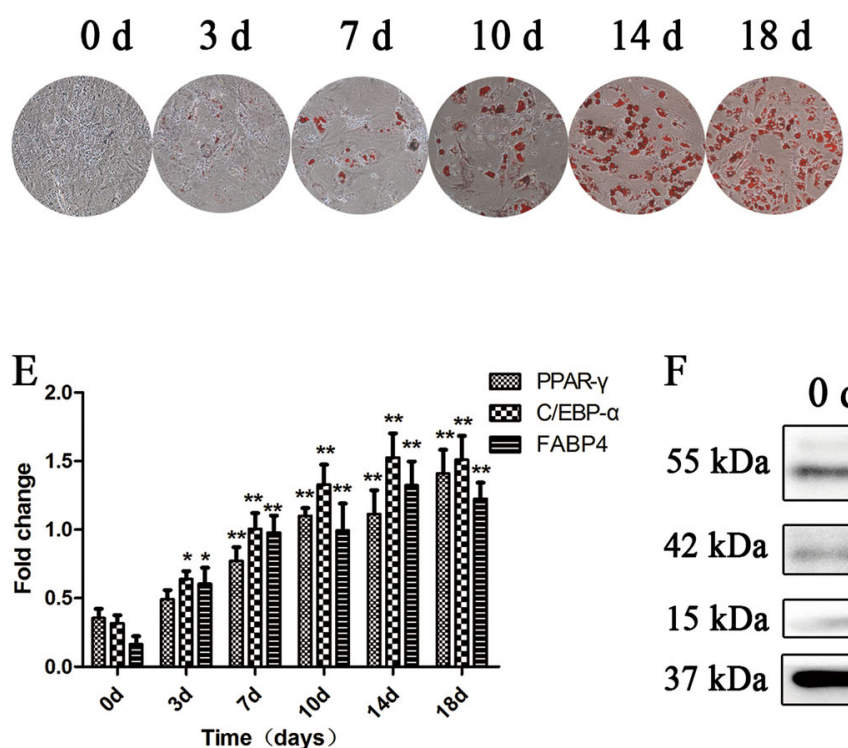

$\mathrm{F}$

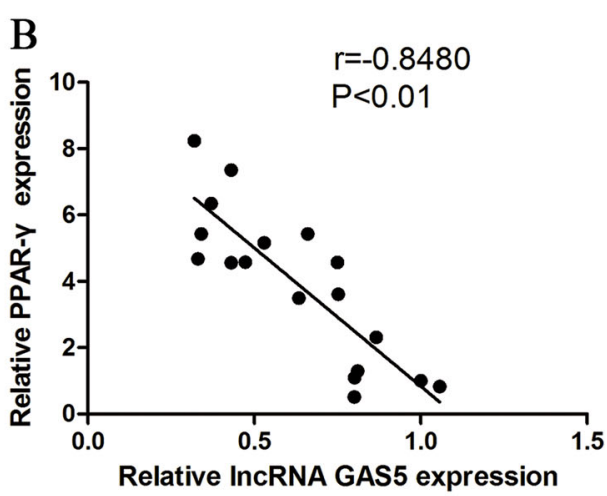

D

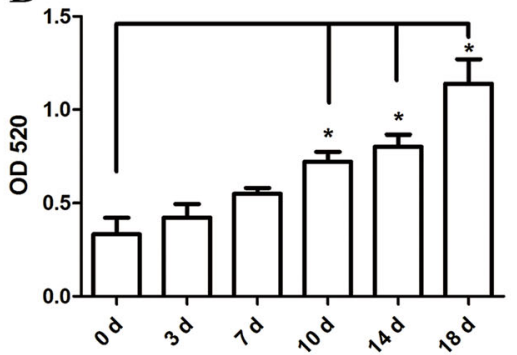

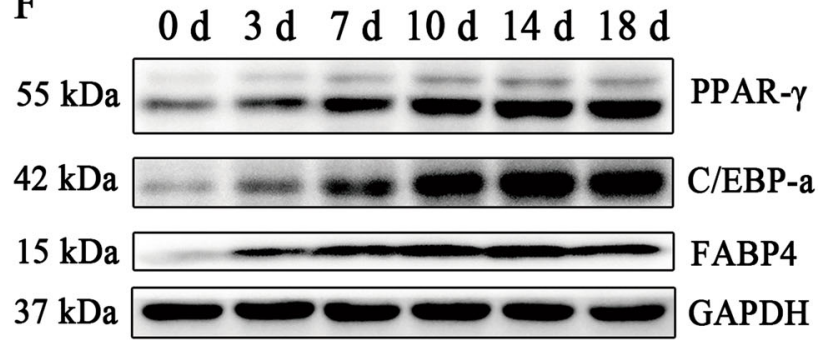

Fig. 1 The IncRNA GAS5 negatively correlates with the adipogenic differentiation capacity of MSCs. a Relative expression of GAS5 during the adipogenic differentiation of MSCs. b Relative expression levels of GAS5 and PPAR- $\gamma$ during the adipogenic differentiation of MSCs obtained from 18 healthy donors. $\mathbf{c}$, $\mathbf{d}$ ORO staining and quantification on days $0,3,7,10,14$, and 18 of MSC adipogenic differentiation. e, $\mathbf{f}$ Relative levels of PPAR- $y$, C/ EBP- $a$ and FABP4 mRNAs and proteins on days $0,3,7,10,14$, and 18 of MSC adipogenic differentiation. The results are presented as the means \pm SD $\left({ }^{*} P<0.05,{ }^{* *} P<0.01\right.$, as determined by Student's $t$-test). All experiments were performed three independent times 


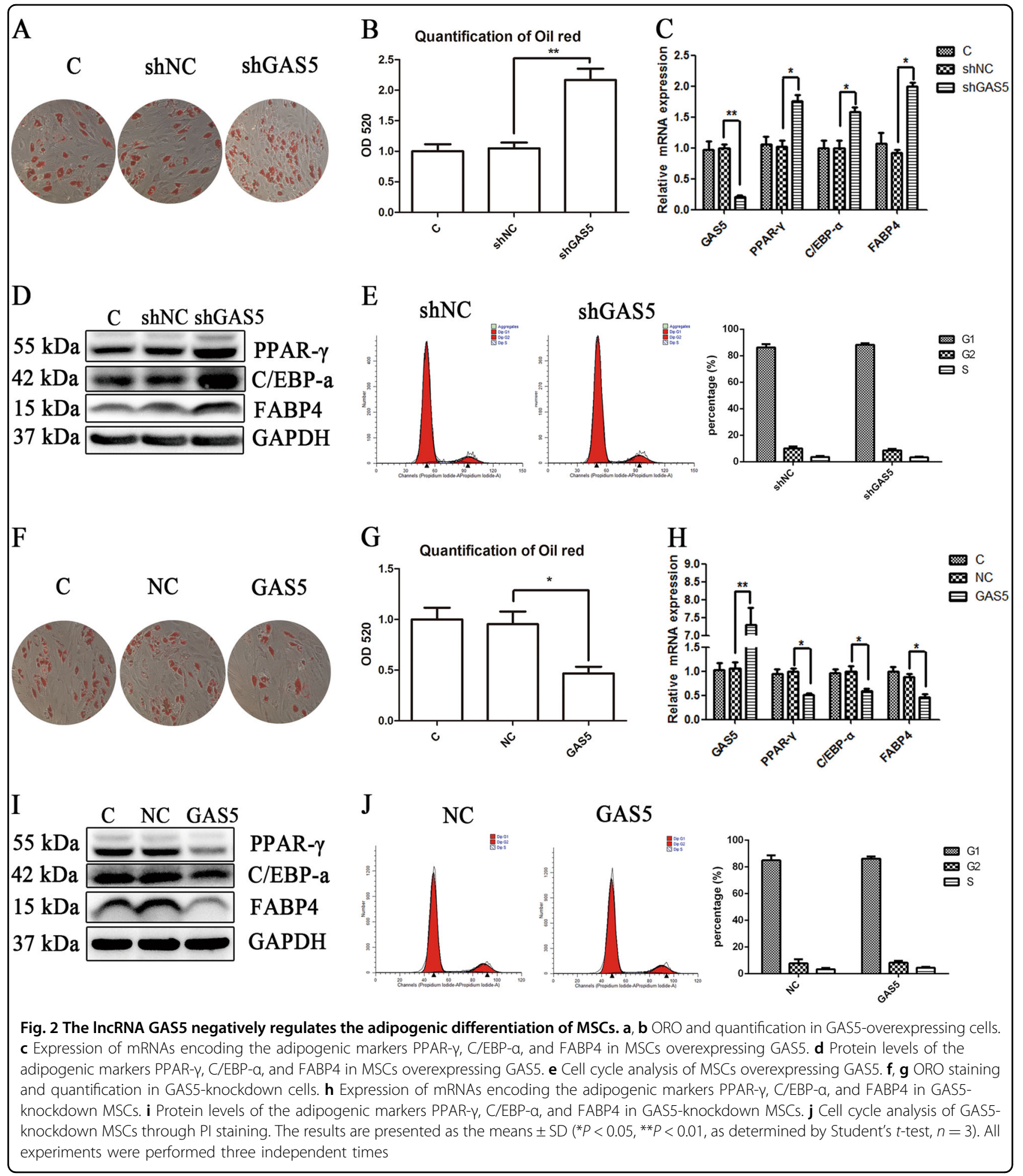

Scientific) and then treated with RNase-free DNase I (Thermo Scientific). The retrieved proteins were detected by Western blotting. The procedure was conducted according to the manufacturer's instructions and standard protocols of Western blot described previously.

\section{Statistical analyses}

The experiments and analyses were separately completed by the different authors listed in this study. Statistical analyses were performed using SPSS 22.0 software (Chicago, IL, USA). The data are expressed as the means 
\pm standard deviations (SD). Differences between two groups were assessed using Student's $t$-test, and differences among three or more groups were analyzed by ANOVA. $P$ values $<0.05$ were considered significant.

\section{Results}

The IncRNA GAS5 negatively correlates with the adipogenic differentiation capacity of MSCs

We characterized the markers of MSCs by flow cytometry and found that the cells in our experiment expressed CD29, CD105, and CD44 but lacked CD45, CD34, and HLA-DR (Supplementary Figure 1A). Alizarin Red S, ORO and toluidine blue staining was performed to detect differentiated cells. The results are shown in Supplementary Figure 1B.

We evaluated the pattern of GAS5 expression by qRTPCR to investigate the impact of this lncRNA on the adipogenic differentiation of MSCs and found that GAS5 expression was downregulated during the adipogenic differentiation of MSCs by approximately $70 \%$ on day 14 (Fig. 1a). We analyzed the relative expression of GAS5 and PPAR- $\gamma$ and observed a negative correlation during the differentiation of MSCs (Fig. 1b), suggesting that GAS5 may exert a negative effect on the differentiation of MSCs into adipocytes. The adipogenic differentiation of MSCs was confirmed by ORO staining, Western blotting and qRT-PCR (Fig. 1c-f).

\section{The IncRNA GAS5 negatively regulates the adipogenic differentiation of MSCs}

We constructed a GAS5-overexpressing lentivirus and infected MSCs to investigate whether the lncRNA GAS5 affects the adipogenic differentiation of MSCs. MSCs were induced toward adipogenic differentiation for 14 days, and ORO staining was performed to determine the effect of GAS5. GAS5 overexpression significantly decreased the formation of adipocytes, according to the ORO staining and quantitative analysis (Fig. 2a, b). The mRNA expression and protein levels of adipogenic markers were decreased in the GAS5 overexpression group (Fig. 2c,d). We subsequently selected the most efficient siRNA among three siRNAs and constructed lentiviruses carrying the shRNA (Supplementary Figure 2A). GAS5 knockdown using the shRNA significantly increased the number of adipogenic of MSCs (Fig. 2f, g) and increased the mRNA and protein expression of PPAR- $\gamma, \mathrm{C} / \mathrm{EBP}-\alpha$ and FABP4 (Fig. 2h, i). We also explored whether GAS5 affected MSC proliferation. The cell cycle of MSCs was not influenced by either GAS5 overexpression or knockdown, as demonstrated by PI staining and the flow
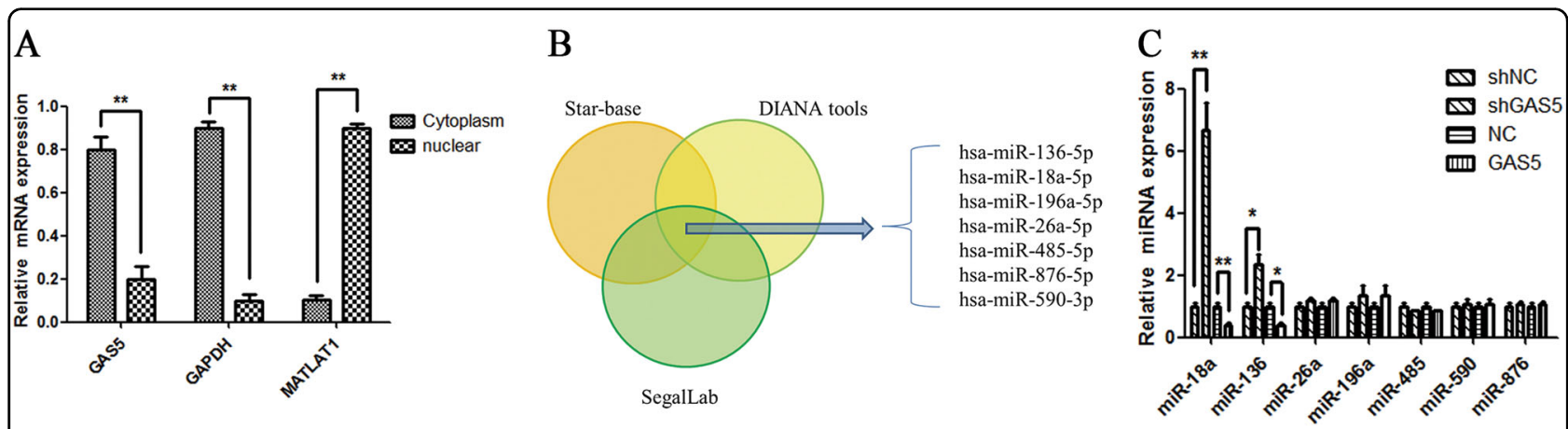

D

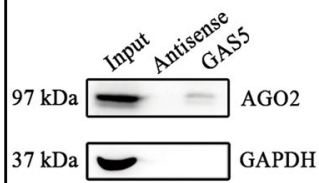

E

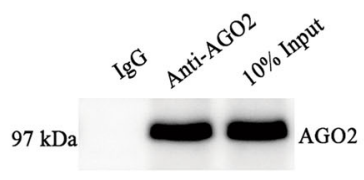

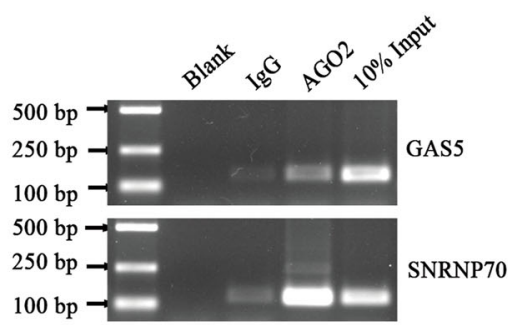

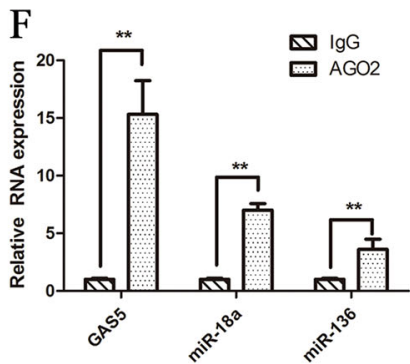

Fig. 3 The IncRNA GAS5 sponges miRNAs as a competing endogenous RNA. a Analysis of GAS5, GAPDH, and MALAT1 levels in the cytoplasmic and nuclear fractions of MSCs. $\mathbf{b}$ Bioinformatics analysis of three databases (ChipBase, LncRNAdb, and StarBase). $\mathbf{c}$ Levels of different miRNAs in GAS5knockdown and GAS5-overexpressing MSCS, as analyzed by qPCR. $\mathbf{d}$ Results of an RNA pull-down assay analyzing the interaction between GAS5 and AGO2. e Results of the RIP assay analyzing the efficiency (left panel) and RT-PCR products of GAS5 and SNRNP70 (right panel). SNRNP70 was used as a positive control in the RIP assay and the immunoprecipitation efficiency analysis. $\mathbf{f}$ Levels of GAS5, miR-18a and miR-136 in the lgG group and the anti-AGO2 group. The results are presented as the means $\pm S D\left({ }^{*} P<0.05,{ }^{* *} P<0.01\right.$, as determined by Student's $t$-test, $\left.n=3\right)$. All experiments were performed three independent times 
cytometry analysis (Fig. 2e, j). Thus, GAS5 negatively regulated the adipogenic differentiation of MSCs.

The IncRNA GAS5 sponges miRNAs by acting as a ceRNA

We isolated cytoplasmic and nuclear fractions from MSCs to identify the specific mechanism through which GAS5 regulates MSC adipogenic differentiation and found that GAS5 was mainly located in the cytoplasm (Fig. 3a). Cytoplasmic lncRNAs have been shown to act as ceRNAs that sponge miRNAs ${ }^{16}$. We performed a bioinformatics analysis of three databases (ChipBase, LncRNAdb, and StarBase) to identify miRNAs that potentially interact with GAS5. The results revealed seven miRNAs with the highest scores for binding to GAS5 (Fig. 3b). We designed qPCR primers for these seven miRNAs to determine whether they were regulated by GAS5. Notably, the levels of miR-18a and miR-136 were significantly increased in the GAS5 knockdown group and decreased in the GAS5 overexpression group (Fig. 3c). We used an RNA pull-down assay to examine whether GAS5 bound to AGO2, the core protein in the RNA-induced silencing complex ${ }^{31}$ (RISC). According to the Western blot results, GAS5, but not the antisense control, bound to AGO2 (Fig. 3d). An RIP assay was performed to further verify the interaction between GAS5 and miR-18a and miR-136. SNRNP70 was used as a positive control in the RIP assay, and the immunoprecipitation efficiency is presented in Fig. 4e. The levels of GAS5, miR-18a, and miR-136 were markedly increased in the anti-AGO2 group compared with the IgG group (Fig. 3f).

\section{GAS5 specifically binds to miRNA-18a to negatively regulate the adipogenic differentiation of MSCs}

We subsequently analyzed the relationship between miR-18a, miR-136 and GAS5, and the results revealed negative correlations for miR-18a (Fig. 4a) and miR-136 (Supplementary Figure 2F) with GAS5. We investigated whether miR-18a influenced the adipogenic differentiation of MSCs. We transfected MSCs with a miR-18a mimic and inhibitor (Fig. 4b), and the resulting adipogenic changes were detected by ORO staining. The miR18a mimic significantly increased the adipogenic differentiation of MSCs (Fig. 4c, d), and this effect was accompanied by changes in the expression of adipogenic markers (Fig. 4e). Based on these results, miR-18a functions in the adipogenic differentiation of MSCs. We also determined whether miR-136 influenced the adipogenic differentiation of MSCs. However, ORO staining and quantification yielded negative results, suggesting that miR-136 is not involved in MSC adipogenic differentiation (Supplementary Figure 2G).

We also checked the possible binding sites for GAS5 in miR-18a and constructed WT and mutation type (MUT) luciferase reporter genes including firefly and Renilla luciferase sequences (Fig. 4f). According to the results of the luciferase assay, the miR-18a mimic significantly decreased the fluorescence of GAS5 WT but had no impact on GAS5 MUT (Fig. 4g). The overexpression of GAS5 WT significantly downregulated miR-18a expression, but GAS5 MUT overexpression in MSCs had no influence on the miR-18a levels (Fig. 4h). Changes in miR18a expression had no influence on GAS5 expression (Supplementary Figure 2B). Thus, GAS5 serves as a sponge of miR-18a, and miR-18a might be the downstream molecular target of GAS5.

We then explored whether miR-18a reversed the effect of GAS5. We co-transfected MSCs with the miR-18a mimic and the GAS5-overexpressing lentivirus and induced adipogenic differentiation for 14 days. ORO staining showed that the miR-18a mimic significantly reversed the effect of GAS5 on decreasing MSC adipogenic differentiation (Fig. 5a, b). Changes in the protein levels of adipogenic markers also supported this reversed effected (Fig. 5c, d). We subsequently explored the effect of the miR-18a inhibitor on GAS5-silenced MSCs. The ORO staining results and the analysis of the PPAR- $\gamma, \mathrm{C} /$ EBP- $\alpha$, and FABP4 protein levels showed that the miR18a inhibitor also reversed the effect of GAS5 knockdown (Fig. 6a-d). In summary, GAS5 inhibits the adipogenic differentiation of MSCs by decreasing the miR-18a levels.

\section{The miR-18a/CTGF axis affects the adipogenic differentiation of MSCs and is modulated by GAS5}

Based on bioinformatics predictions and previous studies, miR-18a functions by binding to the CTGF mRNA. In addition, the levels of the CTGF protein were decreased by the expression of the miR-18a mimic and increased by the expression of the miR-18a inhibitor (Fig. 7a). We examined the level of the CTGF mRNA in MSCs transfected with the miR-18a mimic or inhibitor and obtained negative results (Fig. $7 \mathrm{~b}$ ). The relationship between miR-18a and CTGF is presented in Fig. 7c, which reveals a negative correlation. We also detected the level of the CTGF protein in MSCs transfected by GAS5knockdown or GAS5-overexpression lentiviruses. The level of the CTGF protein was increased by GAS5 overexpression and decreased by shGAS5. The miR-18a mimic or inhibitor reversed the effects of GAS5 overexpression or knockdown, respectively (Fig. 7d, e), indicating that GAS5 affects the adipogenic differentiation of MSCs through a miR-18a/CTGF-dependent mechanism.

We designed three siRNAs to knock down CTGF and determine the effect of CTGF on the adipogenic differentiation of MSCs (Supplementary Figure 2C). We selected siRNA3 (Fig. 7f) to examine whether CTGF knockdown influenced MSC adipogenic differentiation. The CTGF siRNA significantly increased the adipogenic differentiation of MSCs (Fig. 7g, h), and recombinant 


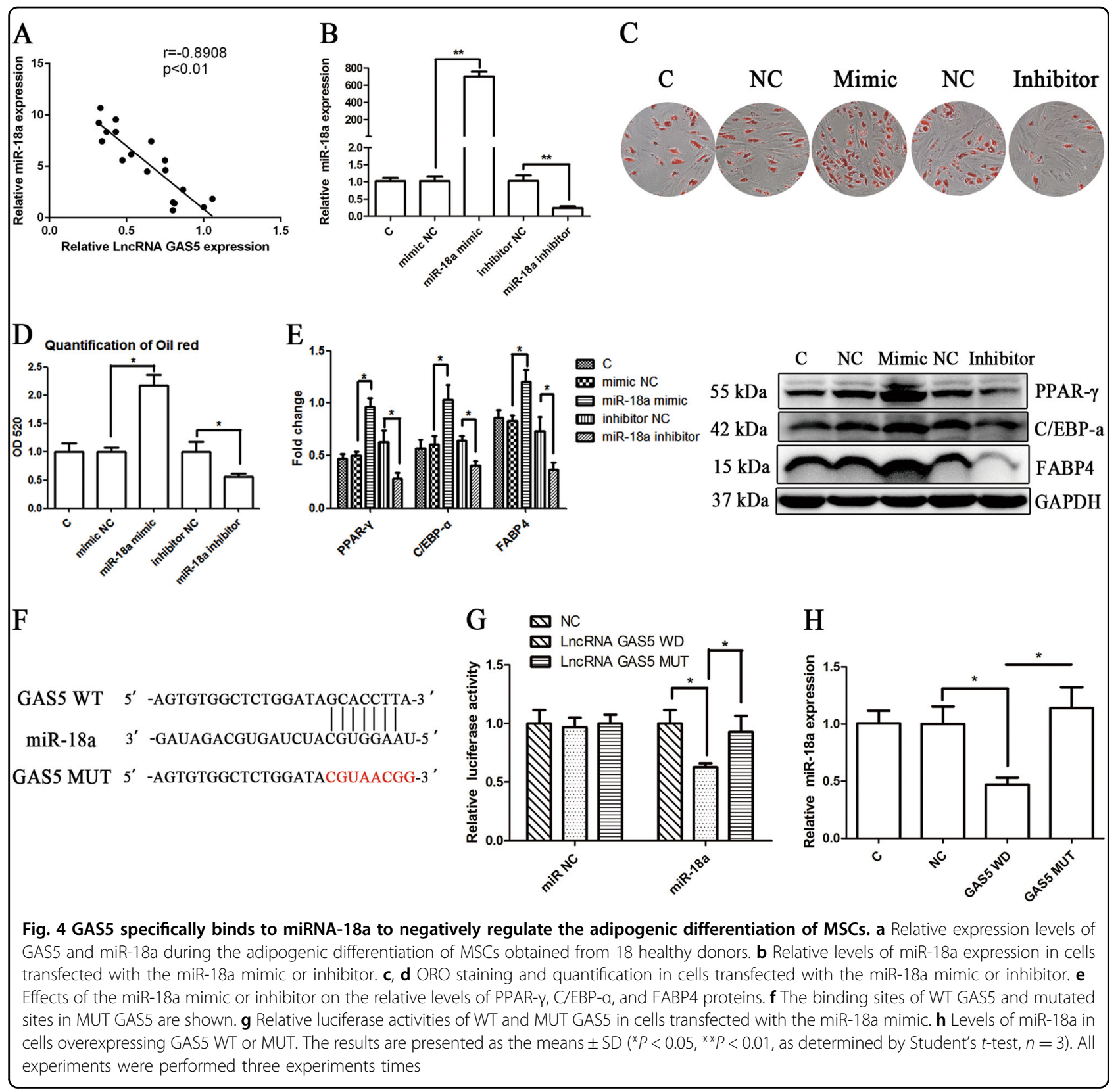

human CTGF (rhCTGF) decreased the adipogenic differentiation of MSCs in a concentration-dependent manner (Fig. 7i, j). The interaction between GAS5, miR18a, and CTGF in MSC adipogenic differentiation is shown in Supplement Fig. 3.

\section{Discussion}

In our study, GAS5 was negatively correlated with the adipogenic differentiation capacity of MSCs. Alterations in GAS5 expression were used to regulate the MSC adipogenic differentiation capability. Based on the results of mechanistic studies, GAS5 acts as a ceRNA that sponges miR-18a. Furthermore, the miR-18a/CTGF axis regulates
MSC adipogenic differentiation. Specifically, GAS5 negatively regulates MSC adipogenic differentiation by modulating the miR-18a/CTGF axis. Therefore, the lncRNA GAS5 negatively regulates the adipogenic differentiation of MSCs by modulating the miR-18a/CTGF axis as a ceRNA.

lncRNAs have been reported to play important roles in many biological functions. For instance, the lncRNA EGFR-AS1 mediates epidermal growth factor receptor addiction and modulates treatment responses in squamous cell carcinoma ${ }^{32}$. DDX5 and its associated lncRNA Rmrp modulate TH17 cell effector functions ${ }^{33}$. lnc-DC binds to STAT3 to influence dendritic cell 
A

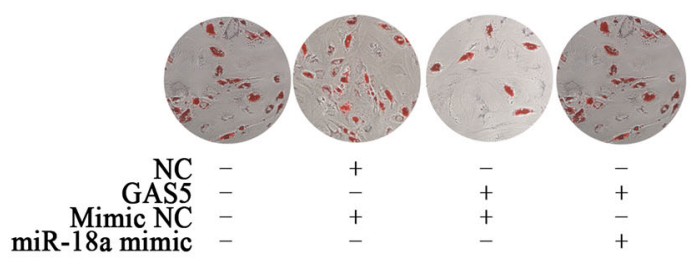

C

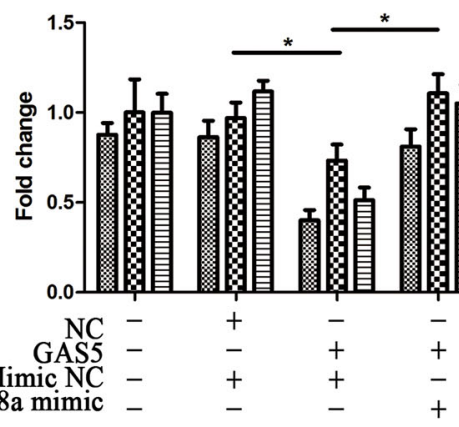

B

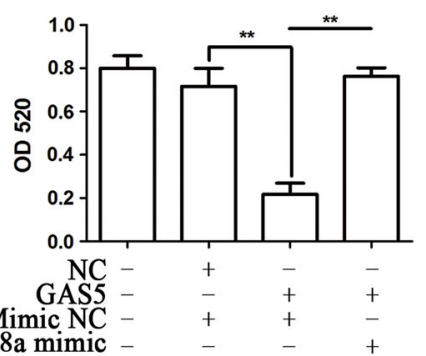

D

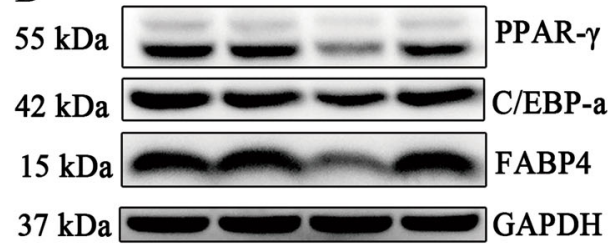

$\begin{array}{llll}- & + & - & - \\ - & - & + & + \\ - & + & + & - \\ - & - & - & +\end{array}$

Fig. 5 The miR-18 mimic reverses the effect of GAS5 overexpression on the adipogenic differentiation of MSCs. a ORO staining in GAS5overexpressing MSCs transfected with the miR-18a mimic. b Quantification of ORO staining in GAS5-overexpressing MSCs transfected with the miR18a mimic. c Quantification of the relative levels of PPAR-y, C/EBP- $a$, and FABP4 proteins in GAS5-overexpressing MSCs transfected with the miR-18a mimic. d Western blots showing the PPAR- $\gamma, C / E B P-a$, and FABP4 levels in GAS5-overexpressing MSCs transfected with the miR-18a mimic. The results are presented as the means \pm SD $\left({ }^{*} P<0.05,{ }^{* *} P<0.01\right.$, as determined by Student's $t$-test, $\left.n=3\right)$. All experiments were performed three independent times

A

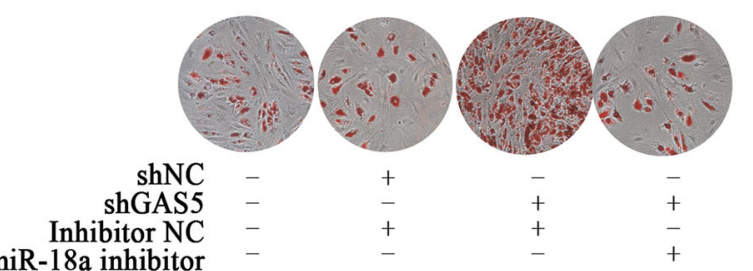

$\mathrm{C}$

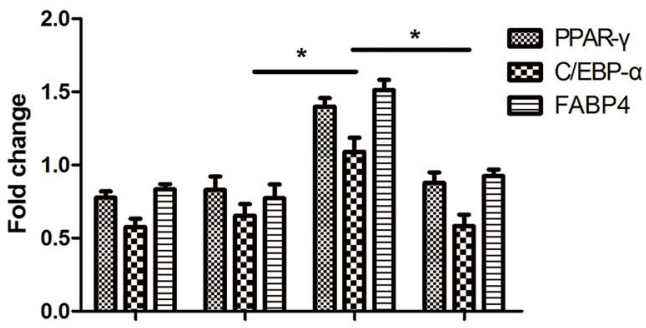

shNC shGAS5 -

Inhibitor NC miR-18a inhibitor

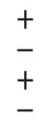

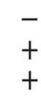

B

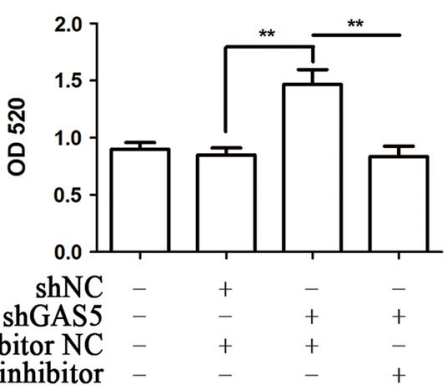

miR-18a inhibitor

D

$55 \mathrm{kDa}$

$42 \mathrm{kDa}$

$15 \mathrm{kDa}$

$37 \mathrm{kDa}$

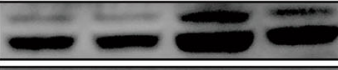

PPAR- $\gamma$

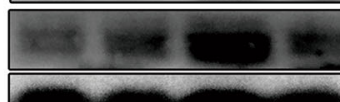

C/EBP-a

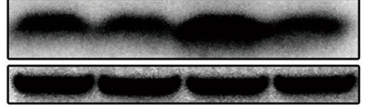

FABP4

shNC

shGAS5

Inhibitor NC

miR-18a inhibitor

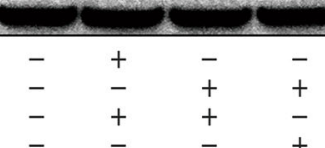

GAPDH

Fig. 6 The miR-18a inhibitor reverses the effect of GAS5 knockdown on the adipogenic differentiation of MSCs. a ORO staining in GAS5knockdown MSCs transfected with the miR-18a inhibitor. b Quantification of ORO staining in GAS5-knockdown MSCs transfected with the miR-18a inhibitor. c Quantification of the relative levels of PPAR- $\gamma$, C/EBP- $a$, and FABP4 proteins in GAS5-knockdown MSCs transfected with the miR-18a inhibitor. d Western blots showing the PPAR- $\gamma$, C/EBP-a, and FABP4 levels in GAS5-knockdown MSCs transfected with the miR-18a inhibitor. The results are presented as the means $\pm S D\left({ }^{*} P<0.05\right.$, ${ }^{*} P<0.01$, as determined by Student's $t$-test, $\left.n=3\right)$. All experiments were performed three independent times 


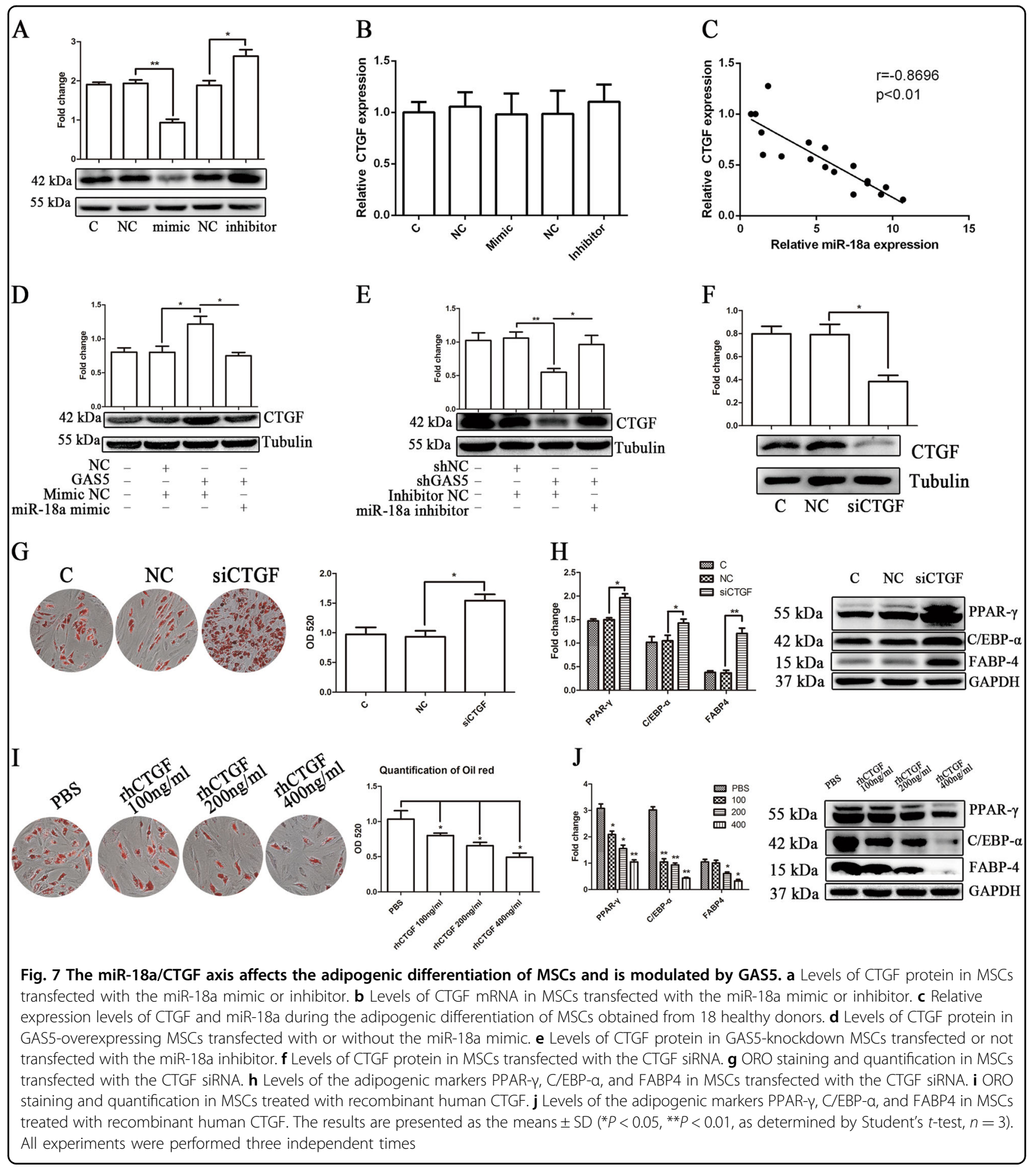

differentiation ${ }^{34}$. The lncRNA GAS5 is considered a cancer suppressor ${ }^{9}$ but was recently shown to be a vital regulator of the self-renewal of human embryonic stem cells $^{35}$. Thus, GAS5 may play an essential role in cell development and differentiation. In the present study, GAS5 was negatively correlated with adipogenic differentiation. The upregulation of GAS5 expression resulted in a corresponding change in adipogenic differentiation, suggesting that GAS5 negatively regulates MSC adipogenic differentiation. GAS5 correlates with tumor growth and invasion ${ }^{8,11}$, and in our previous studies, we found some lncRNAs were associated with abnormal 
pathological osteoblasts in ankylosing spondylitis ${ }^{36}$. Therefore, abnormal expression of the lncRNA GAS5 in MSCs may be linked to various MSC-related diseases.

According to their location in the cell, IncRNAs are divided into two types: nuclear lncRNAs and cytoplasmic lncRNAs. IncRNAs located in the nucleus function by affecting transcription factors or by inducing epigenetic modifications ${ }^{37,38}$, whereas lncRNAs located in the cytoplasm are predominantly competitive endogenous RNAs $^{18,39}$. In the present study, GAS5 was found to be located in the cytoplasm of MSCs, consistent with its location in other cell types ${ }^{18,39}$. GAS5 has been shown to function as a ceRNA, but the mechanism of GAS5 in MSCs has not been reported. Our experiments suggest that GAS5 functions as a ceRNA, consistent with other reports in the literature ${ }^{40,41}$, and affects the adipogenic differentiation of MSCs via the miR-18a/CTGF axis. Recently, some researchers proposed the mechanism of target RNA-directed miRNA degradation (TDMD) ${ }^{42}$ and showed that miRNA degradation is more active in primary neurons. Additionally, TDMD sites appear to be two independent processes, and their balance is regulated by alterations in miRNA abundance. However, the canonical miRNA binding sites neither induce miRNA decay nor compromise TDMD. In our research, GAS5 sponged miR-18a by binding to the canonical binding sites, and the sponge mechanism has been verified in several cell types. This information improves our knowledge of the mechanisms underlying the interactions between lncRNAs and miRNAs. Nevertheless, the sponge mechanism and TDMD appear to be cell-specific processes and deserve further exploration.

MicroRNAs are a class of noncoding single-stranded RNA molecules with a length of approximately 22 nucleotides and encoded by endogenous genes. These molecules are involved in the regulation of posttranscriptional gene expression in cell biology. MicroRNAs, such as miR-140 ${ }^{43}, \operatorname{miR}-27 \mathrm{a}^{14}$, and miR-223 ${ }^{15}$, influence the adipogenic differentiation of MSCs. MicroRNA-18a is an important member of the miR-17-92 cluster, but the role of miR-18a in the differentiation of MSCs has not been evaluated. As shown in the present study, miR-18a promotes the adipogenic differentiation of MSCs. CTGF (CCN2) is a member of the CCN (CYR61, CTGF, and NOV) family of proteins. The effect of miR18a on CTGF was confirmed in previous studies examining human corneal epithelial cells and in pneumoconiosis $^{27,44}$. In addition, CTGF promotes the proliferation and invasion of a variety of tumor cells ${ }^{45}$. A study of CTGF and MSCs by Battula and coworkers found that CTGF is required for bone marrow fat formation after leukemia bone marrow engraftment ${ }^{28}$. In our study, CTGF negatively regulated the adipogenic differentiation of MSCs, whereas CTGF itself was regulated by miR-18a.
Therefore, the miR-18a/CTGF axis plays a vital role in MSC differentiation. As mentioned above, GAS5 functions as a ceRNA, and the experimental results showed that GAS5 negatively regulates the miRNA18/CTGF axis. Thus, GAS5 negatively regulates the adipogenic differentiation of MSCs by modulating the miR-18a/CTGF axis. CTGF expressed in MSCs is an important factor in leukemia and tongue squamous cell carcinoma ${ }^{28,45}$. However, research regarding the functions of GAS5 and miR18a in these diseases is lacking. Our findings may provide insights into the mechanisms through which GAS5 and miR-18a correlate with CTGF in diseases. However, further research is needed to test the specific role of GAS5 in these diseases.

Adipose tissue comprises a substantial amount of biologically active tissues; non-obese men and women have $\sim 12 \mathrm{~kg}$ and $\sim 14 \mathrm{~kg}$ of adipose tissue, respectively ${ }^{46}$. Adipocytes are the basic components of adipose tissue, and MSCs are a main source of adipocytes in many tissues. Normal MSC adipogenic differentiation helps maintain the physiological balance in the body. However, the abnormal adipogenic differentiation of MSCs is associated with many disorders, such as cardiovascular disease ${ }^{47}$, poor glycemic control ${ }^{48}$ and hematopoietic diseases ${ }^{49}$. Thus, an understanding of the mechanisms regulating the adipogenic differentiation of MSCs is of great importance. Based on our findings, the lncRNA GAS5 negatively regulates the adipogenic differentiation of MSCs by modulating the miR-18a/CTGF axis as a ceRNA. This information provides a new theoretical basis for clarifying the mechanism of MSC differentiation and provides a novel therapeutic target for the treatment of adipocyterelated diseases. The adipogenic differentiation of MSCs is currently widely applied for augmentation and reconstruction in aesthetic and reconstructive surgery ${ }^{4,50,51}$. The results of our study will be helpful for expanding the applications of MSCs in the surgical reconstruction field. However, our research has some limitations, such as the lack of an animal model to study the function of GAS5 in vivo. In addition, researchers have not yet determined whether GAS5 affects osteogenic or chondrogenic differentiation, and the specific mechanism through which GAS5 regulates the miR-18a/CTGF axis has not been elucidated. Additional studies are needed to surmount these shortcomings. Nonetheless, GAS5 may play a greater role in the function and application of MSCs in the future.

\footnotetext{
Acknowledgements

The authors thank Yuxiao Ye and American Journal Experts for providing English language editing of the manuscript. This work was supported by grants from the Guangdong Provincial Industrial Technology Research and Development of Special Funds Project (grant number 20160911), Guangzhou Science and Technology Plan (grant number 201604020004), and Guangdong Province Science and Technology Plan (grant number 2015B090903059).
} 


\section{Author details}

1Department of Orthopedics, Sun Yat-sen Memorial Hospital, Sun Yat-sen University, Guangzhou 510120, People's Republic of China. 'Department of Orthopedics, Zhujiang Hospital, Southern Medical University, Guangzhou 510120, People's Republic of China. ${ }^{3}$ Center for Biotherapy, Sun Yat-sen Memorial Hospital, Sun Yat-sen University, Guangzhou 510120, People's Republic of China

\section{Author contributions}

M.L., Z.X., and P.W. designed the study and performed the experiments; J.L., W. L., S.T. performed the experiment, Z.L., X.W., Y.W. analyzed the data; M.L., Z.X., and H.S. wrote the manuscript.

\section{Conflict of interest}

The authors declare that they have no conflict of interest.

\section{Publisher's note}

Springer Nature remains neutral with regard to jurisdictional claims in published maps and institutional affiliations.

Supplementary Information accompanies this paper at https://doi.org/ 10.1038/s41419-018-0627-5.

Received: 1 February 2018 Revised: 19 April 2018 Accepted: 23 April 2018 Published online: 10 May 2018

\section{References}

1. Uccelli, A., Moretta, L. \& Pistoia, V. Mesenchymal stem cells in health and disease. Nat. Rev. Immunol. 8, 726-736 (2008).

2. Trounson, A. \& McDonald, C. Stem cell therapies in clinical trials: progress and challenges. Cell Stem Cell 17, 11-22 (2015).

3. Visscher, L. E. et al. Breast augmentation and reconstruction from a regenerative medicine point of view: state of the art and future perspectives. Tissue Eng. Part B Rev. 23, 281-293 (2017).

4. Kim, I. et al. Clinical implication of allogenic implantation of adipogenic differentiated adipose-derived stem cells. Stem Cells Transl. Med 3, 1312-1321 (2014).

5. Chen, Q. et al. Fate decision of mesenchymal stem cells: adipocytes or osteoblasts? Cell Death Differ. 23, 1128-1139 (2016).

6. Zhang, $X$. et al. Crif1 promotes adipogenic differentiation of bone marrow mesenchymal stem cells after irradiation by modulating the PKAVCREB signaling pathway. Stem Cells 33, 1915-1926 (2015).

7. Kalwa, M. et al. The IncRNA HOTAIR impacts on mesenchymal stem cells via triple helix formation. Nucleic Acids Res. 44, 10631-10643 (2016).

8. Dong, S. et al. The long non-coding RNA, GAS5, enhances gefitinib-induced cell death in innate EGFR tyrosine kinase inhibitor-resistant lung adenocarcinoma cells with wide-type EGFR via downregulation of the IGF-1R expression. J. Hematol. Oncol. 8, 43 (2015).

9. Zhao, X. et al. Gas5 exerts tumor-suppressive functions in human glioma cells by targeting miR-222. Mol. Ther. 23, 1899-1911 (2015).

10. Mourtada-Maarabouni, M., Hedge, V. L., Kirkham, L., Farzaneh, F. \& Williams, G. T. Growth arrest in human T-cells is controlled by the noncoding RNA growth-arrest-specific transcript 5 (GAS5). J. Cell Sci. 121, 939-946 (2008)

11. Kino, T., Hurt, D. E., Ichiijo, T., Nader, N. \& Chrousos, G. P. Noncoding RNA gas5 is a growth arrest- and starvation-associated repressor of the glucocorticoid receptor. Sci. Signal 3, ra8 (2010).

12. Luo, G. et al. LncRNA GAS5 inhibits cellular proliferation by targeting P27(Kip1). Mol. Cancer Res. 15, 789-799 (2017)

13. Bartel, D. P. MicroRNAs: target recognition and regulatory functions. Cell $\mathbf{1 3 6}$ 215-233 (2009).

14. You, L., Pan, L., Chen, L., Gu, W. \& Chen, J. MiR-27a is essential for the shift from osteogenic differentiation to adipogenic differentiation of mesenchymal stem cells in postmenopausal osteoporosis. Cell Physiol. Biochem 39, 253-265 (2016).

15. Guan, X. et al. miR-223 regulates adipogenic and osteogenic differentiation of mesenchymal stem cells through a C/EBPs/miR-223/FGFR2 regulatory feedback loop. Stem Cells 33, 1589-1600 (2015).
16. Grimson, A. Noncoding RNA: linking microRNAs to their targets. Nat. Chem Biol. 11, 100-101 (2015).

17. Militello, G. et al. Screening and validation of IncRNAs and circRNAs as miRNA sponges. Brief. Bioinform. 18, 780-788 (2017).

18. Yan, $\mathrm{H}$. et al. Long non-coding RNA MEG3 functions as a competing endogenous RNA to regulate ischemic neuronal death by targeting miR-21/ PDCD4 signaling pathway. Cell Death Dis. 8, 3211 (2017).

19. Song, Y. et al. MiR-18a regulates the proliferation, migration and invasion of human glioblastoma cell by targeting neogenin. Exp. Cell Res. 324, 54-64 (2014).

20. Liang, C. et al. MicroRNA-18a-5p functions as an oncogene by directly targeting IRF2 in lung cancer. Cell Death Dis. 8, e2764 (2017).

21. Liu, L., Cai, X., Liu, E., Tian, X. \& Tian, C. MicroRNA-18a promotes proliferation and metastasis in hepatocellular carcinoma via targeting KLF4. Oncotarget 8, 68263-68269 (2017).

22. Kee, H. J. et al. miR-18a-5p microRNA increases vascular smooth muscle cell differentiation by downregulating syndecan4. Korean Circ. J. 44, 255-263 (2014).

23. Hong, $X$. et al. Phenanthrene-induced apoptosis and its underlying mechanism. Environ. Sci. Technol. 51, 14397-14405 (2017).

24. Cheung, L. C. et al. Connective tissue growth factor is expressed in bone marrow stromal cells and promotes interleukin-7-dependent B lymphopoiesis. Haematologica 99, 1149-1156 (2014).

25. Luo, Q. et al. Connective tissue growth factor (CTGF) is regulated by Wnt and bone morphogenetic proteins signaling in osteoblast differentiation of mesenchymal stem cells. J. Biol. Chem. 279, 55958-55968 (2004).

26. Tomita, N. et al. Cartilage-specific over-expression of CCN family member 2/ connective tissue growth factor (CCN2/CTGF) stimulates insulin-like growth factor expression and bone growth. PLOS ONE 8, e59226 (2013).

27. Guo, Y., Lu, X. \& Wang, H. Downregulation of miR-18a induces CTGF and promotes proliferation and migration of sodium hyaluronate treated human corneal epithelial cells. Gene 591, 129-136 (2016).

28. Battula, V. L. et al. Connective tissue growth factor regulates adipocyte differentiation of mesenchymal stromal cells and facilitates leukemia bone marrow engraftment. Blood 122, 357-366 (2013).

29. Wang, P. et al. Effects and safety of allogenic mesenchymal stem cell intravenous infusion in active ankylosing spondylitis patients who failed NSAIDs: a 20-week clinical trial. Cell Transplant. 23, 1293-1303 (2014).

30. Li, Y. et al. Whole genome expression profiling and signal pathway screening of MSCs in Ankylosing Spondylitis. Stem Cells Int. 2014, 913050 (2014).

31. Pasquinelli, A. E. MicroRNAs and their targets: recognition, regulation and an emerging reciprocal relationship. Nat. Rev. Genet. 13, 271-282 (2012).

32. Tan, D. S. W. et al. Long noncoding RNA EGFR-AS1 mediates epidermal growth factor receptor addiction and modulates treatment response in squamous cell carcinoma. Nat. Med. 23, 1167-1175 (2017).

33. Huang, W. et al. DDX5 and its associated IncRNA Rmrp modulate TH17 cell effector functions. Nature 528, 517-522 (2015).

34. Wang, P. et al. The STAT3-binding long noncoding RNA Inc-DC controls human dendritic cell differentiation. Science 344, 310-313 (2014).

35. $\mathrm{Xu}, \mathrm{C}$. et al. Long non-coding RNA GAS5 controls human embryonic stem cell self-renewal by maintaining NODAL signalling. Nat. Commun. 7 13287 (2016).

36. Xie, Z. et al. Differential expression profiles of long noncoding RNA and mRNA of osteogenically differentiated mesenchymal stem cells in ankylosing spondylitis. J. Rheumatol. 43, 1523-1531 (2016).

37. Chen, Z. Z. et al. LncSox4 promotes the self-renewal of liver tumour-initiating cells through Stat3-mediated Sox4 expression. Nat. Commun. 7, 12598 (2016).

38. Wang, $Z$. et al. The long noncoding RNA Chaer defines an epigenetic checkpoint in cardiac hypertrophy. Nat. Med. 22, 1131-1139 (2016).

39. Wang, G., Pan, J., Zhang, L., Wei, Y., Wang, C. Long non-coding RNA CRNDE sponges miR-384 to promote proliferation and metastasis of pancreatic cancer cells through upregulating IRS1. Cell Prolif, 50, e12389 (2017).

40. Gao, Z. Q. et al. Long non-coding RNA GAS5 suppresses pancreatic cancer metastasis through modulating miR-32-5p/PTEN axis. Cell Biosci. 7, 66 (2017).

41. Zhang, Z. et al. Negative regulation of IncRNA GAS5 by miR-21. Cell Death Differ. 20, 1558-1568 (2013).

42. de la Mata, M. et al. Potent degradation of neuronal miRNAs induced by highly complementary targets. EMBO Rep. 16, 500-511 (2015).

43. Gernapudi, R. et al. MicroRNA 140 promotes expression of long noncoding RNA NEAT1 in adipogenesis. Mol. Cell Biol. 36, 30-38 (2016). 
44. Yang, $\mathrm{H}$. et al. Regulatory role of miR-18a to CCN2 by TGF-beta1 signaling pathway in pulmonary injury induced by nano-SiO, Environ. Sci. Pollut. Res. Int. 25, 867-876 (2018).

45. $\mathrm{Wu}, \mathrm{Y}$. L. et al. Mesenchymal stem cell-derived CCN2 promotes the proliferation, migration and invasion of human tongue squamous cell carcinoma cells. Cancer Sci. 108, 897-909 (2017).

46. Parker, R., Kim, S. J. \& Gao, B. Alcohol, adipose tissue and liver disease: mechanistic links and clinical considerations. Nat. Rev. Gastroenterol. Hepatol. 15, 50-59 (2018).

47. Fox, C. S. et al. Abdominal visceral and subcutaneous adipose tissue compartments: association with metabolic risk factors in the Framingham Heart Study. Circulation 116, 39-48 (2007).
48. Ross, R., Fortier, L. \& Hudson, R. Separate associations between visceral and subcutaneous adipose tissue distribution, insulin and glucose levels in obese women. Diabetes Care 19, 1404-1411 (1996).

49. Ambrosi, T. H. et al. Adipocyte accumulation in the bone marrow during obesity and aging impairs stem cell-based hematopoietic and bone regeneration. Cell Stem Cell 20, 771-784.e776 (2017)

50. Sinna, R., Delay, E., Garson, S. \& Mojallal, A. Scientific bases of fat transfer Critical review of the literature. Ann. Chir. Plast. Esthet. 51, 223-230 (2006).

51. Caplan, A. I. \& Correa, D. The MSC: an injury drugstore. Cell Stem Cell 9, 11-15 (2011). 